\title{
Morphology, Temperature, and Field-Dependence of Charge Separation in High-Efficiency Solar Cells Based on Alternating Polyquinoxaline Copolymer
}

\author{
Artem A. Bakulin ${ }^{1,2}$, Yuxin Xia, ${ }^{3}$ Huib J. Bakker ${ }^{1}$, Olle Inganäs ${ }^{3}$, Feng Gao ${ }^{3 *}$ \\ ${ }^{1}$ FOM Institute AMOLF, Science Park 104, Amsterdam, The Netherlands \\ ${ }^{2}$ Cavendish Laboratory, University of Cambridge, JJ Thomson Ave, Cambridge CB30HE, UK \\ ${ }^{3}$ Biomolecular and Organic Electronics, IFM, Linköping University, Linköping 58183, Sweden \\ *Corresponding authors: aab58@cam.ac.uk;fenga@ifm.liu.se
}

\begin{abstract}
Charge separation and recombination are key processes determining the performance of organic optoelectronic devices. Here we combine photoluminescence and photovoltaic characterisation of organic solar cell devices with ultrafast multi-pulse photocurrent spectroscopy to investigate charge generation mechanisms in the organic photovoltaic devices based on a blend of an alternating polyquinoxaline copolymer with fullerene. The combined use of these techniques enables the determination of the contributions of geminate and bimolecular processes to the solar cell performance. We observe that charge separation is not a temperature-activated process in the studied materials. At the same time, the generation of free charges shows a clear external-field and morphology dependence. This indicates that the critical step of charge separation involves the non-equilibrium state that is formed at early times after photoexcitation, when the polaronic localisation is not yet complete. This work reveals new aspects of molecular level charge dynamics in the organic light-conversion systems.
\end{abstract}




\section{Introduction:}

Plastic solar cells, using a mixture of organic (macro)molecules as active element, hold the potential to yield a low-cost photovoltaic technology. ${ }^{1-4}$ Due to the solution processability of organic molecular systems, cost-efficient manufacturing techniques such as roll-to-roll processing or ink-jet printing can be applied. ${ }^{5}$ While the efficiency of plastic photovoltaic devices is constantly improving ${ }^{6}$ with internal quantum efficiencies approaching and even exceeding $90 \%$, the fundamental mechanism of free charge carrier generation in organic semiconducting materials is still under debate. ${ }^{7-28}$

In organic semiconductors the photoexcited electron remains strongly bound to the hole in the valence band thus forming a stable neutral excitonic state. The spontaneous dissociation probability of such an exciton into a charge pair is low. To enhance charge separation, for example for photovoltaic applications, heterojunctions of different materials are used. The differences in the energy levels of the molecular orbitals of these materials provide the driving potential for electron and hole separation. Recently it has become clear that long-range charge separation is a complex multistep process. After the initial electron transfer an intermolecular, so called, charge transfer (CT) state is composed of an electrostatically bound electron-hole pair with a very different dissociation probability in different molecular systems. The properties of CT states have been investigated in a range of experimental studies ${ }^{12,14,29-37}$ addressing different aspects of CT phenomenon. These studies have spawned a variety of models explaining the process of electron and hole detachment in organic heterojunction systems. Initially, these models were semi-classical based on (i) the thermodynamic balance between the lowest CT state and 'free' separated charges (SC) energy levels, usually referred to as 'cold' dissociation 9,23 and (ii) a kinetic model of the competition between thermalisation and dissociation ${ }^{17-19,38}$ referred to as 'hot' dissociation. An enchancement of the local mobility ${ }^{39-41}$ or dissociation during cooling (due to energetic disorder) has also been proposed to explain the efficient separation of charges. ${ }^{42}$ In parallel, a number of quantum mechanical models have been proposed in which the electronic dynamics is determined by delocalization ${ }^{15,24,43}$ and vibronic 44-47 effects.

Each of the above charge-separation models relates to a specific dependence of the charge separation efficiency on temperature, local morphologies and external field. This has triggered a number of studies where the dissociation/recombination yield of CT states was investigated as a function of several parameters. Both time-resolved and steady-state measurements showed that for almost all organic material systems geminate CT state 
dissociation is independent ${ }^{8,9,20,23}$ or very weakly dependent ${ }^{48}$ on temperature. On the opposite, the local morphology, particularly ordering and aggregation effects, seem to play a critical role for the efficiency of long-range charge separation. ${ }^{24,49}$ There is less consensus on the field dependence of charge separation. While most ultrafast (sub-ns) studies reported minor effects of the applied electric field, ${ }^{24}$ experiments on longer time scales demonstrated both strong and weak field influences on the charge separation. ${ }^{16,50,51}$ We note that this discrepancy can be explained from the fact that $\mathrm{CT}$ states can be generated directly from excitonic states and result from bimolecular recombination of separated charge carriers. Time-integrated measurements like CT PL cannot distinguish between those two pathways and would highly benefit from being complemented with time-resolved techniques.

This contribution addresses the photophysics of charge separation in poly[2,3-bis-(3octyloxyphenyl)quinoxaline-5,8-diyl-alt-thiophene-2,5-diyl] (TQ1) : [6,6]-phenyl-C 71 -butyric acid methyl ester ( $\left.\mathrm{PC}_{71} \mathrm{BM}\right)$ (fig.1a) bulk heterojunction solar cells. These solar cells provide efficiencies up to $6 \%{ }^{52}$, and therefore represent a promising molecular system for the development of organic photovoltaics. We have investigated the morphology, temperature and external field dependence of these devices using CT PL, photocurrent, and pump-push photocurrent (PPP) spectroscopy. The combination of these three techniques allowed for a careful separation of geminate and bimolecular phenomena as well as crosschecks for the spectroscopic model (fig.1b) derived. We show that TQ1:PC ${ }_{71} \mathrm{BM}$ blends display temperatureindependent but strongly morphology-dependent charge generation, similar to what has been observed for other polymer fullerene systems. However, quite surprisingly, the quantum yield of CT relaxation/dissociation is observed to depend strongly on the applied electric field dependent in TQ1:PC ${ }_{71} \mathrm{BM}$. The overall data set indicates that the early (sub-ps) charge dynamics, when the molecular system is far from equilibrium and polaronic reorganisation did not occur yet, is critical for charge separation in the studied material.

\section{Experimental:}

Materials and devices. TQ1 was synthesized according to previously published procedures, ${ }^{52}$ and $\mathrm{PC}_{71} \mathrm{BM}$ was purchased from Solenne Netherlands. For device preparation, PEDOT:PSS layer $(40 \mathrm{~nm}$ ) was spin-coated onto cleaned ITO (indium-tin-oxide) substrates, which were subsequently annealed at $120^{\circ} \mathrm{C}$ for 30 minutes before being transferred into a glovebox for further fabrication. TQ1 and $\mathrm{PC}_{71} \mathrm{BM}$ were dissolved in orthodichlorobenzene (oDCB) with a polymer concentration of $10 \mathrm{mg} / \mathrm{ml}$. TQ1:PC ${ }_{71} \mathrm{BM}$ solutions were spin-coated onto the substrates to get films with a thickness of $\sim 100 \mathrm{~nm}$. We evaporated layers of $0.6 \mathrm{~nm}$ 
of $\mathrm{LiF}$ and $100 \mathrm{~nm}$ of aluminum onto the active layer under high vacuum $\left(10^{-6} \mathrm{mbar}\right)$ conditions, and we used a shadow mask to define the active area of $\sim 4.5 \mathrm{~mm}^{2}$.

The AFM characterization of the film surfaces (see Supplementary Information) indicated morphology typical for similar polymer:fullerene material systems. At low $\mathrm{PC}_{71} \mathrm{BM}$ loading a homogeneous mixture is formed. At high concentration, signatures of fullerene crystallites are observed which are expected to improve long-range charge separation.

PL measurements under external bias. The devices were mounted in a liquid-nitrogen cryostat and were kept in an atmosphere of low-pressure ( 10 mbar) nitrogen during the measurements. PL emission spectra of the devices were recorded from the ITO side using an Oriel liquid light guide and a Shamrock SR 303i spectrograph coupled to a Newton EMCCD silicon detector. The transmission of the measuring system was radiometrically calibrated using an Optronic OL245 M standard spectral irradiance lamp. A blue CW 405 nm PMM-208G-VT laser with an intensity of $4 \mathrm{~mW}$ was employed to excite the samples from the ITO side. The bias was applied using a Keithley 2400 Source Measure Unit.

Pump-push photocurrent spectroscopy. A regenerative $1 \mathrm{kHz}$ Ti:Sapphire amplifier system (Coherent, Legend Elite Duo) was used to pump a broadband non-collinear optical amplifier (Clark) and a 2-stage home-built optical parametric amplifier (OPA) to generate 100fs visible pump pulses ( $\sim 40 \mathrm{~nm}$ central wavelength, $\pm 20 \mathrm{~nm}$ bandwidth) and 70-fs infrared push pulses $(2000 \pm 100 \mathrm{~nm})$, respectively. We focused $\sim 1 \mathrm{~nJ}$ visible pump and $\sim 1 \mu \mathrm{J}$ infrared push pulses onto a $\sim 1 \mathrm{~mm}^{2}$ spot on the device. The reference photocurrent from a photodiode was detected at a pump repetition frequency of $1 \mathrm{kHz}$ using a lock-in amplifier. The push beam was mechanically chopped at $\sim 370 \mathrm{~Hz}$, and its effect on the photocurrent was also detected by a lock-in amplifier. The push pulse also induces a minor current in the device by itself due to absorption from sub-gap states and long-lived trapped charges. This current component does not depend on the delay with respect to the visible pump pulse and was subtracted from the presented measurements. In field-dependent PPP measurements the external bias was applied by a $9 \mathrm{~V}$ battery with a dividing resistor to minimize the voltage source noise. 

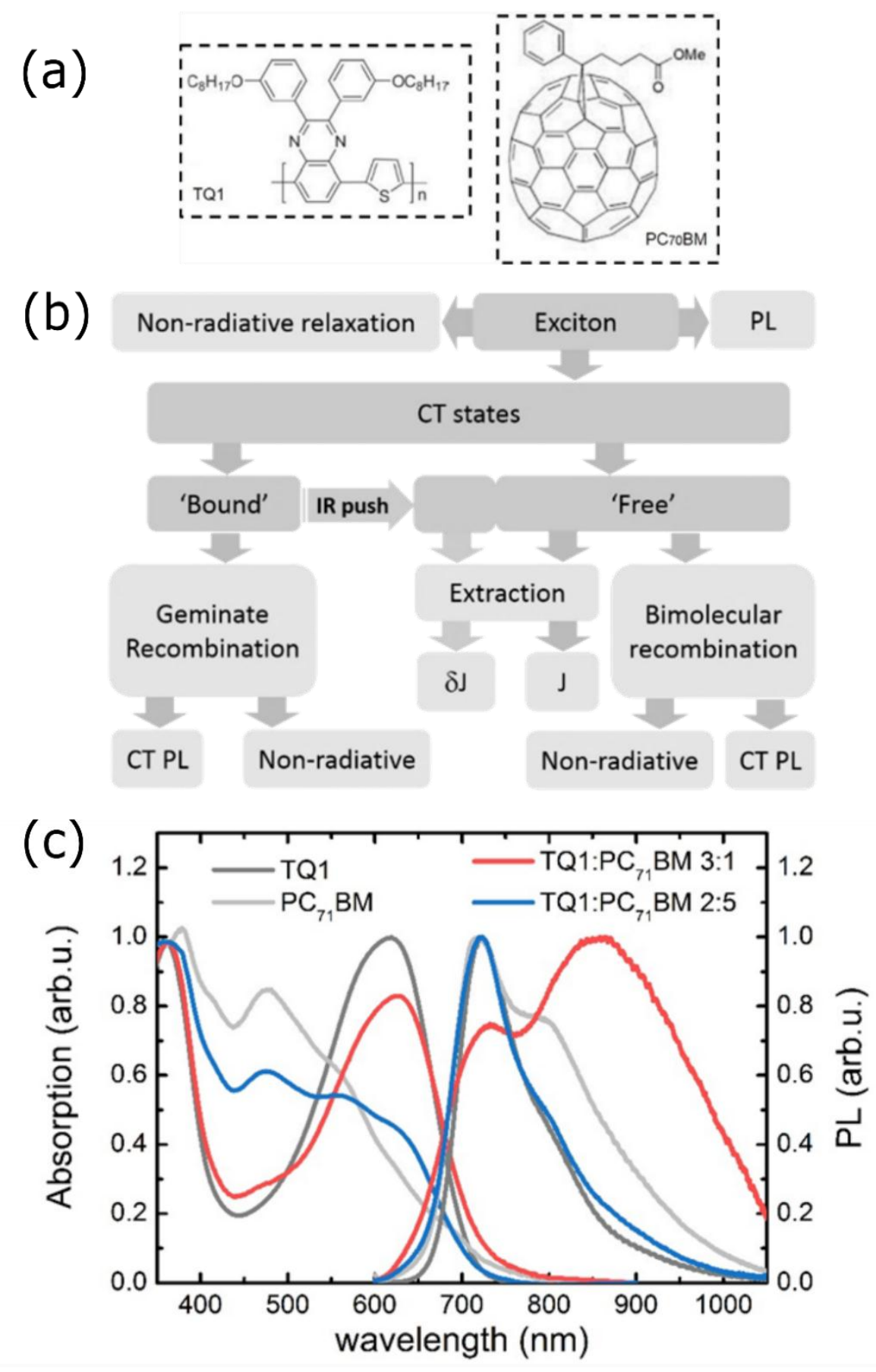

Figure 1. (a) Structural formulas of the materials under study; (b) The diagram describing CT state relaxation and the experimental observables of this study to elucidate the CT dynamics (c) Absorption and PL spectra of TQ1, PC $C_{71} B M$ and their blends.

\section{Results:}

Figure 1a presents the structural formulas of the used materials. Figure 1c shows absorption and photoluminescence (PL) spectra of the pure TQ1 and $\mathrm{PC}_{71} \mathrm{BM}$ and of the blends used in this study.The peaks at 710-780 nm in the PL emission correspond to the overlapping PL emission bands of TQ1 and $\mathrm{PC}_{71} \mathrm{BM}$. The peak at $880 \mathrm{~nm}$ appears only in the blends and is 
associated with the emission from intermolecular CT states. The CT PL contribution can be deconvoluted from the total PL spectrum with a reasonable accuracy, which makes this contribution a sensitive probe for carrier recombination occurring at the donor-acceptor interface. ${ }^{53}$ The relative amplitude of the CT PL emission depends on the acceptor concentration which demonstrates that the charge separation in $\mathrm{TQ} 1: \mathrm{PC}_{71} \mathrm{BM}$ blends has a clear composition and morphology dependence. Same time, the actual energy of CT state when estimated from the peak of CT PL emission was observed to be the same for different morphologies and biases within $0.03 \mathrm{eV}$ error bar. The shift of CT PL emission peak with temperature did not exceed $0.05 \mathrm{eV}$ in agreement with previous studies. ${ }^{54}$

To study the device-relevant photophysics of TQ1:PC ${ }_{71} \mathrm{BM}$ bulk heterojunctions in detail, we fabricated a set of photovoltaic devices and investigated these using CT PL, photocurrent and PPP spectroscopic methods. In addition to the morphology/composition variation we also varied the temperature and the applied external field. Below we first address composition, temperature, and field dependences separately and then discuss all the results together to elucidate the charge generation mechanism TQ1:PC ${ }_{71} \mathrm{BM}$ molecular system.

\section{Morphology effect}

Figure 2a illustrates the photovoltaic characterization of OPVs. The two devices show a significant difference in short-circuit current $\left(J_{s c}\right)$ and fill factor (FF). When increasing the $\mathrm{PC}_{71} \mathrm{BM}$ concentration from $25 \%$ ( $3: 1$ blend) to $\sim 70 \%$ (2:5 blend), $J_{s c}$ increases from 0.96 to $8.8 \mathrm{~mA} / \mathrm{cm}^{2}$, and the FF increases from $23 \%$ to $57 \%$. The increase in the efficiency correlates well with the decrease of the CT PL emission as shown in figure $2 \mathrm{~b}$. Based on previous studies, ${ }^{24}$ we associate the acceptor concentration dependence of the photovoltaic performance and charge separation with the modification of the local morphology. It has been found that with increasing amount of $\mathrm{PC}_{71} \mathrm{BM} \sim 10-50 \mathrm{~nm}$ acceptor domains ${ }^{55}$ are formed in the active layer, which is highly favorable for the overall device performance. The effect of morphology may have two possible origins. The quantum efficiency may be increased due to (i) a more efficient charge separation promoted by the delocalization of carriers ${ }^{24,43}$ or (ii) an improved charge transport through the device. The observed change in the PL emission from the CT states as a function of material composition points at the former mechanism. On the other hand, CT PL may also occur as a result of bimolecular recombination of non-geminate pairs. Hence, the decrease in CT PL may also be due to non-geminate recombination associated with a better transport of the electrons and holes through the device. 
(a)

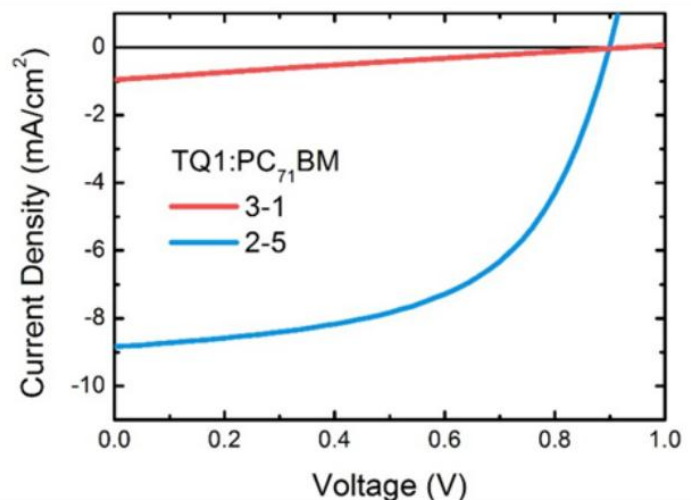

(b)
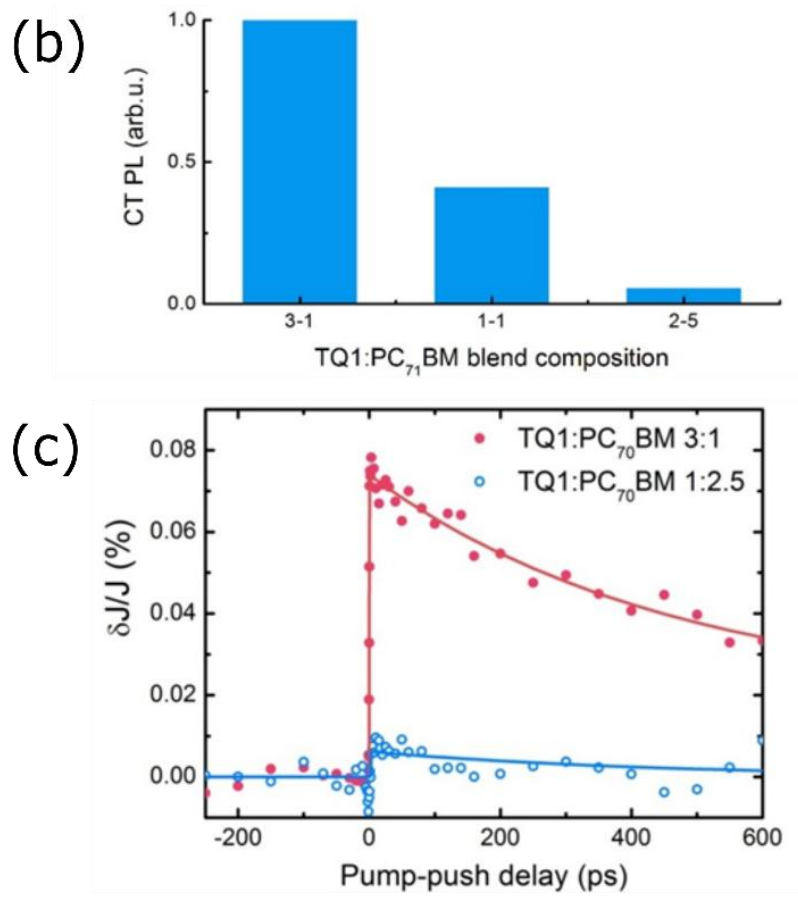

Figure 2. (a) I-V characteristics for the devices with different relative concentration of TQI and PC $C_{71} B M$. (b) The amplitude of CT PL for devices with a different TQ1:PC $71 B M$ concentrations. (c) PPP transients for TQ1:PCBM 3:1 and 2:5 devices at room temperature and short-circuit conditions. Solid lines are exponential guides-to-the-eye convoluted with the experimental setup response function.

To identify the early-time dynamics of CT states we use pump-push photocurrent (PPP) spectroscopy. ${ }^{56}$ This optoelectronic method was initially developed in the visible spectral region by Frankevich and co-workers to study charge dissociation in organic semiconductors ${ }^{57}$, and was recently extended ${ }^{58}$ to the infrared ${ }^{10}$ and applied to new classes of materials ${ }^{49,59,60}$. The PPP technique measures the amount of 'bound' charge carriers (e.g. bound CT states) as a function of time delay with respect to their generation. Normally the bound charge carriers do not contribute to the photocurrent and they vanish due to the geminate recombination but, with 
the help of the extra energy provided by the IR push pulse, the bound charges are (re)activated and converted to free charge carriers. Thereby they can contribute to the photocurrent (fig.1b).

The ratio of the IR-induced current $\delta J$ and the normally (without push) extracted current $J$, defines the ratio between bound and free charges in the device:

$\frac{\delta J}{J}(V)=\frac{P_{\text {extraction }}(V) \times\left(N_{\text {bound }}(V) \times P_{\text {activation }}\right)}{P_{\text {extraction }}(V) \times N_{\text {free }}(V)}=P_{\text {activation }} \times \frac{N_{\text {bound }}}{N_{\text {free }}}(V) \sim \frac{N_{\text {bound }}}{N_{\text {free }}}(V)$

Where $N_{\text {bound }}$ and $N_{\text {free }}$ are the concentrations of bound and free carriers, $P_{\text {activation }}$ is the probability to activate a bound carrier (e.g.to dissociate a bound CT state) with the IR pulse, and $P_{\text {extraction }}$ is the probability for a free charge to escape bimolecular recombination and to be collected at the electrode.

Figure 2c shows PPP transients measured for TQ1:PC 71 BM 3:1 and 2:5 devices at room temperature and at short-circuit conditions. For both material compositions, the effect of the push pulse builds-up fast (within $\sim 200$ fs time resolution) at time zero, which is a clear indication of the bound CT state formation happening at the ultrafast time scale as observed by others before. ${ }^{61}$ After this, the PPP response decays on a time scale of $\sim 500 \mathrm{ps,} \mathrm{which} \mathrm{we} \mathrm{assign}$ with the loss of bound CT states. Because this process occurs at the fast (sub-ns) timescale and does not depend on the pump we associate it with the geminate recombination of photogenerated species. ${ }^{21}$ Within the experimental accuracy, the $\mathrm{CT}$ generation/relaxation rates were similar for both blends. However, the relative amount of bound CT states is $\sim 10$ times higher for the 3:1 blend. This difference is similar to the increase in $J_{s c}$ upon increasing the $\mathrm{PC}_{71} \mathrm{BM}$ concentration and qualitatively agrees with the photocurrent and CT PL data. Both correlations show that $\mathrm{CT}$ states form an important loss mechanism in TQ1:PC ${ }_{71} \mathrm{BM}$ devices. To obtain more quantitative information on the recombination pathways in the device we have studied the field-dependence of charge separation process using CT PL measurements, steadystate photocurrent, and ultrafast PPP spectroscopy of CT state dynamics.

\section{External field effect}

Figures $3 \mathrm{a}$ and $3 \mathrm{~b}$ present steady-state CT PL as a function of the applied external bias for TQ1:PC ${ }_{71} \mathrm{BM}$ devices of different composition. For all devices the application of a negative bias leads to a suppression of the CT PL signal, indicative of a suppression of the recombination probability of CT states. The decay is much steeper for the fullerene-rich blend, which points towards a lower CT binding energy in phase-segregated material. However, again there are two different scenarios that can explain the bias-dependent CT PL quenching. The first is the dissociation of geminate CT states by the external field immediately after photoexcitation. The 
second, is the field enhanced charge transport and extraction, which would decrease bimolecular regeneration and the recombination of $\mathrm{CT}$ states at longer time scales. To discriminate between those two effects and to acquire additional information about the geminate CT state dynamics, we performed PPP experiments on the devices under external bias.

Figure 3c presents the results of PPP measurements on a TQ1:PC ${ }_{71} \mathrm{BM}$ 3:1 solar cell as a function of applied bias. Both the amplitude and the time evolution of the signal changes with the external voltage, which indicates that the yield of bound CT states and their geminate recombination time are effected by the external field. This agrees with the CT PL results and indicates that the bias decreases the early-time recombination probability in TQ1:PC ${ }_{71} \mathrm{BM}$. We note that in the most efficient material at longer times we observe minor negative PPP effect which we previously associated with push-induced bimolecular recombination. ${ }^{10}$ For this reason only the maximum value of short-term kinetics will be used for analysis below. To quantitatively analyze the field dependence of the PPP and CT PL signals, equation (1) can be rewritten under the assumption of field/independent IR activation:

$P_{\text {activation }}(V)=$ const

In this case, the results of PPP experiments can be directly compared to the results of steady state CT PL and the photocurrent measurements. Assuming that the probability for emissive CT state relaxation $\sigma_{P L}$ does not depend on the external field, one can write:

$\frac{P L_{C T}}{J_{c w}}(V)=\frac{\sigma_{P L} \times N_{\text {bound }}(V)}{P_{\text {extraction }}(V) \times N_{\text {free }}(V)} \sim P_{\text {extraction }}(V)^{-1} \frac{N_{\text {bound }}}{N_{\text {free }}}(V)$

In case the field-dependent charge extraction (and bimolecular recombination) would play a significant role in the photophysics, the right hand parts of equations (1) and (3) will not be equal. However, if the main loss mechanism is geminate CT state relaxation, the extraction efficiency will not play a big role and can be presumed field independent $P_{\text {extraction }}(V)=$ const. In this case combining (1), (2), and (3) we obtain:

$\frac{P L_{C T}}{J_{c w}}(V) \sim \frac{N_{\text {bound }}}{N_{\text {free }}}(V) \sim \frac{\delta J}{J}(V)$

Equation (4) provides a simple experimental criterion for identifying the origin of CT PL. When it is fulfilled, the observed CT PL emission mostly results from geminate recombination. This is also an indication that $\mathrm{CT}$ state formation and relaxation is the dominant loss channel limiting the device performance. Charge extraction in this case is field independent and relatively efficient; therefore, bimolecular recombination does not form a major 
contribution to the current losses. If (4) is not fulfilled, long time charge dynamics, transport and bimolecular recombination constitute the dominant loss channel.
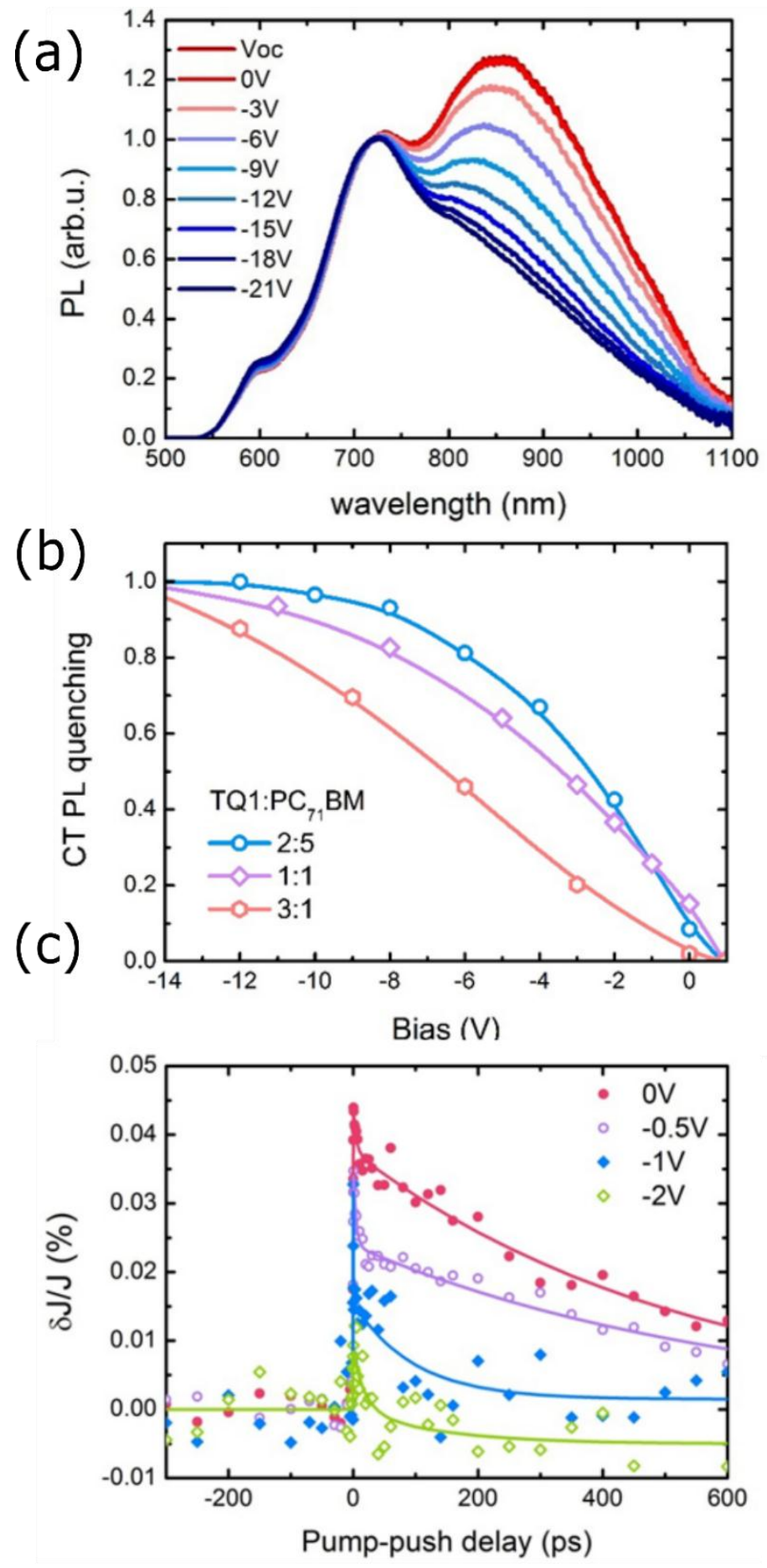

Figure 3. (a) Field-dependent PL in a TQ1:PC ${ }_{71} B M$ 3:1 device. (b) Field-dependent CT PL in TQ1:PC ${ }_{71} B M$ devices of different donor-acceptor composition. (c) Pump-push photocurrent transients for TQ1:PC ${ }_{71} B M$ 3:1 solar cell at different external biases applied. Solid lines are multi-exponential guides to the eye after convolution with the setup response function. 
Figure 4 applies the criterion (4) to the steady-state PL, photocurrent and PPP experimental results for a TQ1:PC ${ }_{71} \mathrm{BM} 3: 1$ photovoltaic device. We note that number of bound CT states in 2:5 blend is too low (see figure.2c) to perform field dependent measurement on this material system. The blue circles represent the CT PL to photocurrent ratio for TQ1:PC ${ }_{71} \mathrm{BM} 3: 1$, while the red crosses represent the amplitude of the corresponding PPP transients. The two parameters demonstrate a very similar external bias dependence, which quantitatively confirms that the geminate recombination of $\mathrm{CT}$ states forms the major loss channel in the studied system. There are two more conclusions to be drawn from these results. First, the observation that charge extraction and bimolecular recombination does not limit the efficiency in 3:1 TQ1:PC $\mathrm{P}_{71} \mathrm{BM}$ blend can be generalised also to the TQ1:PC ${ }_{71} \mathrm{BM}$ blend compositions with higher acceptor concentrations and with better morphologies. It is well known that in a majority of polymer/fullerene blends, ${ }^{24}$ including TQ1:PC ${ }_{71} \mathrm{BM},{ }^{52}$ an increase of the acceptor fraction has a positive effect on the local morphology and charge extraction. Second, steady state CT PL in TQ1:PC ${ }_{71} \mathrm{BM}$ probes mostly the geminate recombination of CT states and has a negligible contribution from bimolecular CT recombination. Based on these results, we studied the temperature dependence of the charge separation only with steady-state methods applied to the high efficiency 2:5 device only.

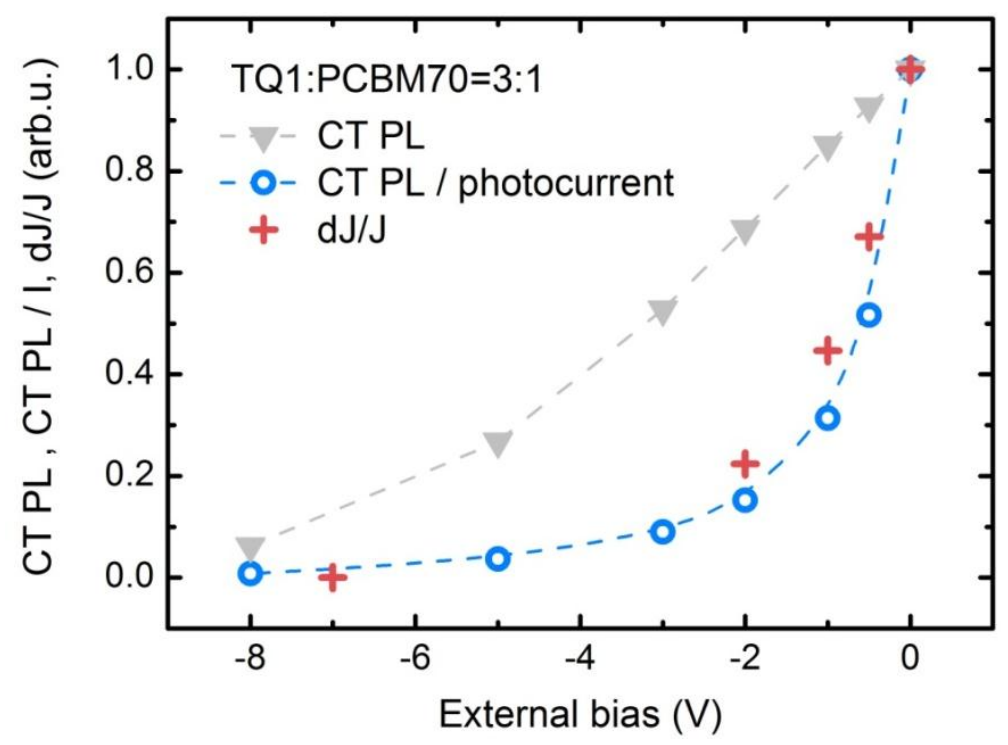

Figure 4. The ratio between the recombined and free charges as measured by CT PL and pumppush spectroscopy. The match between steady-state and PPP observables evidences that CT PL is representative for geminate charge recombination 


\section{Temperature effect}

Figure 5a shows the CT PL signal as a function of bias for a TQ1:PC ${ }_{71} \mathrm{BM}$ 2:5 device at different temperatures. It is clear that the temperature dependencies are very similar which indicates that the molecular mechanism of charge separation does not change with temperature. This result is consistent with our recent finding that for efficient polymer:fullerene blends (including the blend $\mathrm{TQ1}: \mathrm{PC}_{71} \mathrm{BM} 2: 5$ ), charge separation is efficient above cryogenic temperatures. ${ }^{62}$ Very similar behaviour was observed for TQ1:PC ${ }_{71} \mathrm{BM}$ 3:1 material system (see Supplementary Information) confirming that mechanism of charge separation in it is very similar. The independence of charge separation on temperature in this blend provides strong evidence that the influence of the external field is most important at early times after the photoexcitation, when CT state thermalisation is not yet completed.

Figure $5 b$ shows the steady state photocurrent as a function of bias in the same $2: 5$ device at different temperatures. In contrast to CT PL, the photocurrent dependence is clearly getting less steep when the temperature goes down. This implies that charge extraction and bimolecular recombination are much more temperature-dependent processes than charge separation. It is likely that the extraction becomes the performance-limiting process at low $<190 \mathrm{~K}$ temperatures, when the initial CT state dissociation is still very efficient.

\section{Discussion}

By combining steady-state photoluminescence, electrical characterisation and ultrafast opticalphotocurrent spectroscopy, we demonstrate that charge separation in TQ1:PC ${ }_{71} \mathrm{BM}$ OPVs is highly morphology- and field-dependent, but temperature-independent (above cryogenic temperatures). The field dependence of the charge separation has been debated, due to difficulties in disentangling field-dependent charge separation from charge extraction. Here we compare the ratio between the recombined and free charges as measured by CT PL and pumppush spectroscopy. The good match between the steady-state and PPP observables provides strong evidence that CT PL is representative for geminate charge recombination, and thus we demonstrate that the electric field strongly affects charge separation in the studied systems. 

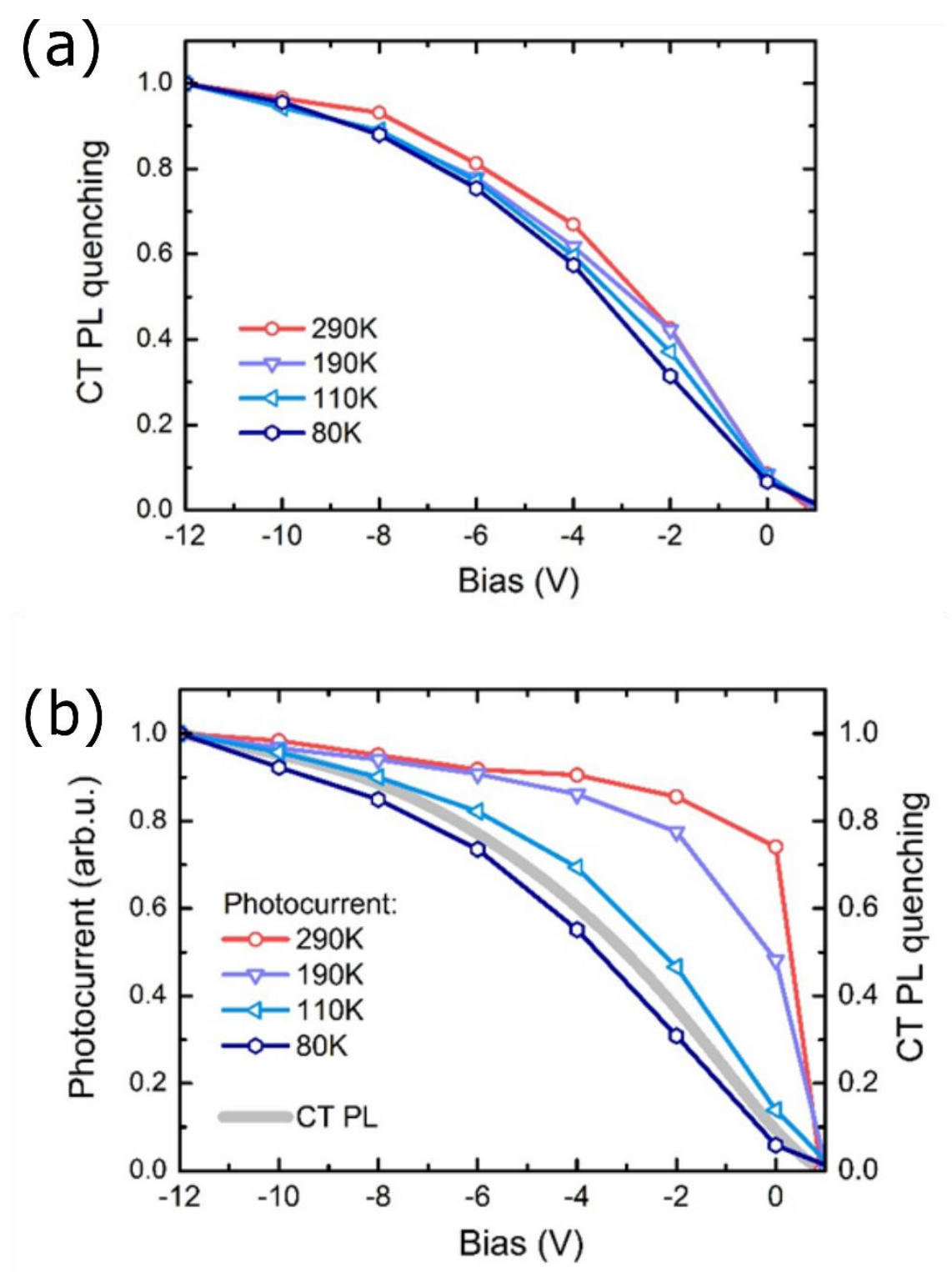

Figure 5. (a) The field dependence of CT PL quenching at different temperatures for the TQ1:PC ${ }_{71} B M$ 2:5 blend. (b) The field dependence of steady state photocurrent at different temperature for TQ1:PC ${ }_{71} B M$ 2:5 blend. Grey solid curve is an average of CT PL quenching dependences panel (a). All curves are normalised to the maximum value reached at $-12 \mathrm{~V}$.

Since temperature has a negligible effect on charge separation in a wide temperature range (from $80 \mathrm{~K}$ to room temperature), the influence of the external field and morphology on charge separation is most important at early stages of charge dynamics, when CT state thermalization has not yet occurred. This conclusion is further confirmed by pump-push spectroscopy, where both morphology- and field-dependent PPP signals indicate that the push effect is relevant at the time scale of 0.1-100 ps. Our results question the thermodynamic model, that proposes that there is a balance between lowest CT state and separated charges. Instead, the results agree well 
with both the dissociation during cooling model ${ }^{42}$ and the quantum mechanical model ${ }^{24,41}$, that emphasize the importance of energetic disorder or charge delocalization in the charge separation process. With increasing fullerene content (from the 3:1 to the 2:5), an increasing amount of charge carriers delocalizes in the fullerene clusters, contributing to the enhanced device performance.

\section{Conclusions}

We combined photoluminescence and electrical characterisation of organic photovoltaic devices with ultrafast optical-photocurrent spectroscopy to investigate the charge generation in $\mathrm{TQ1}: \mathrm{PC}_{71} \mathrm{BM}$ material. Applying this range of techniques to the same set of devices at similar experimental conditions allowed us to disentangle the influences of geminate and bimolecular recombination to the device performance. We observe that charge separation in these materials shows a strong external-field and morphology dependence, and little temperature dependence. This indicates that the critical step of charge separation takes place at early times after photoexcitation when the system is far from equilibrium and polaronic reorganisation has not yet occurred. While the observed behaviour is specific for $\mathrm{TQ} 1: \mathrm{PC}_{71} \mathrm{BM}$ solar cells, it is likely illustrative for molecular level charge dynamics in organic light-conversion systems.

\section{Acknowledgements}

We thank Maxim Pschenichnikov for useful discussions, and Ergang Wang for providing TQ1. This work was supported by the Netherlands Organization for Scientific Research (NWO) through the "Stichting voor Fundamenteel Onderzoek der Materie" (FOM). A.A.B. also acknowledges a VENI grant from NWO. A.A.B. is currently a Royal Society University Research Fellow. Photovoltaics research at Linköping was supported by the Swedish Research Council (VR), the European Commission Marie Skłodowska-Curie actions, the Swedish Energy Agency, and the Knut and Alice Wallenberg foundation (KAW).

Supporting Information Available: morphology characterization, temperature dependence of CT PL for 3:1 TQ1:PC ${ }_{71} \mathrm{BM}$. This material is available free of charge via the Internet http://pubs.acs.org. 


\section{References}

(1) Thompson, B. C.; Frechet, J. M. J. Polymer-Fullerene Composite Solar Cells. Angew. Chem. Int. Ed. 2008, 47, 58-77.

(2) Facchetti, A. Polymer Donor-Polymer Acceptor (All-Polymer) Solar Cells. Mater. Today 2013, 16, 123-132.

(3) Deibel, C.; Dyakonov, V. Polymer-Fullerene Bulk Heterojunction Solar Cells. Rep. Prog. Phys. 2010, 73, 096401.

(4) Li, G.; Zhu, R.; Yang, Y. Polymer Solar Cells. Nat. Photon. 2012, 6, 153-161.

(5) Espinosa, N.; García-Valverde, R.; Urbina, A.; Krebs, F. C. A Life Cycle Analysis of Polymer Solar Cell Modules Prepared Using Roll-to-Roll Methods under Ambient Conditions. Sol. Energy Mater. Sol. Cells 2011, 95, 1293-1302.

(6) Ameri, T.; Li, N.; Brabec, C. J. Highly Efficient Organic Tandem Solar Cells: A Follow up Review. Energy Environ. Sci. 2013, 6, 2390-2413.

(7) Bredas, J.-L.; Norton, J. E.; Cornil, J.; Coropceanu, V. Molecular Understanding of Organic Solar Cells: The Challenges. Acc. Chem. Res. 2009, 42, 1691-1699.

(8) Pensack, R. D.; Asbury, J. B. Barrierless Free Carrier Formation in an Organic Photovoltaic Material Measured with Ultrafast Vibrational Spectroscopy. J. Am. Chem. Soc. 2009, $131,15986-15987$.

(9) Lee, J.; Vandewal, K.; Yost, S. R.; Bahlke, M. E.; Goris, L.; Baldo, M. A.; Manca, J. V.; Voorhis, T. V. Charge Transfer State Versus Hot Exciton Dissociation in Polymer/Fullerene Blended Solar Cells. J. Am. Chem. Soc. 2010, 132, 11878-11880.

(10) Bakulin, A. A.; Rao, A.; Pavelyev, V. G.; van Loosdrecht, P. H. M.; Pshenichnikov, M. S.; Niedzialek, D.; Cornil, J.; Beljonne, D.; Friend, R. H. The Role of Driving Energy and Delocalized States for Charge Separation in Organic Semiconductors. Science 2012, 335, 1340-1344.

(11) Etzold, F.; Howard, I. A.; Mauer, R.; Meister, M.; Kim, T.-D.; Lee, K.-S.; Baek, N. S.; Laquai, F. Ultrafast Exciton Dissociation Followed by Nongeminate Charge Recombination in Pcdtbt:Pcbm Photovoltaic Blends. J. Am. Chem. Soc. 2011, 133, 9469-9479.

(12) Singh, S.; Pandit, B.; Basel, T. P.; Li, S.; Laird, D.; Vardeny, Z. V. Two-Step Charge Photogeneration Dynamics in Polymer/Fullerene Blends for Photovoltaic Applications. Phys. Rev. $B$ 2012, 85, 205206.

(13) Kendrick, M. J.; Neunzert, A.; Payne, M. M.; Purushothaman, B.; Rose, B. D.; Anthony, J. E.; Haley, M. M.; Ostroverkhova, O. Formation of the Donor-Acceptor Charge-Transfer Exciton and Its Contribution to Charge Photogeneration and Recombination in Small-Molecule Bulk Heterojunctions. J. Phys. Chem. C 2012, 116, 18108-18116.

(14) Piliego, C.; Loi, M. A. Charge Transfer State in Highly Efficient Polymer-Fullerene Bulk Heterojunction Solar Cells. J. Mat. Chem. 2012, 22, 4141-4150.

(15) Murthy, D. H. K.; Gao, M.; Vermeulen, M. J. W.; Siebbeles, L. D. A.; Savenije, T. J. Mechanism of Mobile Charge Carrier Generation in Blends of Conjugated Polymers and Fullerenes: Significance of Charge Delocalization and Excess Free Energy. J. Phys. Chem. C 2012, 116, 9214-9220.

(16) Albrecht, S.; Schindler, W.; Kurpiers, J.; Kniepert, J.; Blakesley, J. C.; Dumsch, I.; Allard, S.; Fostiropoulos, K.; Scherf, U.; Neher, D. On the Field Dependence of Free Charge Carrier Generation and Recombination in Blends of Pcpdtbt/Pc70bm: Influence of Solvent Additives. J. Phys. Chem. Lett. 2012, 3, 640-645.

(17) Grancini, G.; Maiuri, M.; Fazzi, D.; Petrozza, A.; Egelhaaf, H. J.; Brida, D.; Cerullo, G.; Lanzani, G. Hot Exciton Dissociation in Polymer Solar Cells. Nat. Mater. 2012, 12, 29-33.

(18) Jamieson, F. C.; Domingo, E. B.; McCarthy-Ward, T.; Heeney, M.; Stingelin, N.;

Durrant, J. R. Fullerene Crystallisation as a Key Driver of Charge Separation in Polymer/Fullerene Bulk Heterojunction Solar Cells. Chem. Sci. 2012, 3, 485-492.

(19) Jailaubekov, A. E.; Willard, A. P.; Tritsch, J. R.; Chan, W.-L.; Sai, N.; Gearba, R.; Kaake, L. G.; Williams, K. J.; Leung, K.; Rossky, P. J.et al. Hot Charge-Transfer Excitons Set the Time Limit for 
Charge Separation at Donor/Acceptor Interfaces in Organic Photovoltaics. Nat. Mater. 2013, 12, 6673.

(20) Dimitrov, S. D.; Bakulin, A. A.; Nielsen, C. B.; Schroeder, B. C.; Du, J.; Bronstein, H.; McCulloch, I.; Friend, R. H.; Durrant, J. R. On the Energetic Dependence of Charge Separation in LowBand-Gap Polymer/Fullerene Blends. J. Am. Chem. Soc. 2012, 134, 18189-18192.

(21) Bakulin, A. A.; Dimitrov, S. D.; Rao, A.; Chow, P. C. Y.; Nielsen, C. B.; Schroeder, B. C.; McCulloch, I.; Bakker, H. J.; Durrant, J. R.; Friend, R. H. Charge-Transfer State Dynamics Following Hole and Electron Transfer in Organic Photovoltaic Devices. J. Phys. Chem. Lett. 2012, 4, 209-215.

(22) Tamura, H.; Burghardt, I. Ultrafast Charge Separation in Organic Photovoltaics Enhanced by Charge Delocalization and Vibronically Hot Exciton Dissociation. J. Am. Chem. Soc. 2013, $135,16364-16367$.

(23) Vandewal, K.; Albrecht, S.; Hoke, E. T.; Graham, K. R.; Widmer, J.; Douglas, J. D.; Schubert, M.; Mateker, W. R.; Bloking, J. T.; Burkhard, G. F.et al. Efficient Charge Generation by Relaxed Charge-Transfer States at Organic Interfaces. Nat. Mater. 2014, 13, 63-68.

(24) Savoie, B. M.; Rao, A.; Bakulin, A. A.; Gélinas, S.; Movaghar, B.; Friend, R. H.; Marks, T. J.; Ratner, M. A. Unequal Partnership: Asymmetric Roles of Polymeric Donor and Fullerene Acceptor in Generating Free Charge. J. Am. Chem. Soc. 2014, 136, 2876-2884.

(25) Gao, F.; Inganas, O. Charge Generation in Polymer-Fullerene Bulk-Heterojunction Solar Cells. Phys. Chem. Chem. Phys. 2014, 16, 20291-20304.

(26) Devižis, A.; De Jonghe-Risse, J.; Hany, R.; Nüesch, F.; Jenatsch, S.; Gulbinas, V.; Moser, J.-E. Dissociation of Charge Transfer States and Carrier Separation in Bilayer Organic Solar Cells: A Time-Resolved Electroabsorption Spectroscopy Study. J. Am. Chem. Soc. 2015, 137, 8192-8198.

(27) Scarongella, M.; De Jonghe-Risse, J.; Buchaca-Domingo, E.; Causa', M.; Fei, Z.; Heeney, M.; Moser, J.-E.; Stingelin, N.; Banerji, N. A Close Look at Charge Generation in Polymer:Fullerene Blends with Microstructure Control. J. Am. Chem. Soc. 2015, 137, 2908-2918.

(28) Bassler, H.; Kohler, A. "Hot or Cold": How Do Charge Transfer States at the DonorAcceptor Interface of an Organic Solar Cell Dissociate? Phys. Chem. Chem. Phys. 2015, 17, 2845128462.

(29) Morteani, A. C.; Sreearunothai, P.; Herz, L. M.; Friend, R. H.; Silva, C. Exciton Regeneration at Polymeric Semiconductor Heterojunctions. Phys. Rev. Lett. 2004, 92, 247402.

(30) Bakulin, A. A.; Khodarev, A. N.; Martyanov, D. S.; Elizarov, S. G.; Golovnin, I. V.; Paraschuk, D. Y.; Arnautov, S. A.; Nechvolodova, E. M. Charge Transfer Complexes of a Conjugated Polymer. Dokl. Chem. 2004, 398, 204-206.

(31) Hwang, I.-W.; Soci, C.; Moses, D.; Zhu, Z.; Waller, D.; Gaudiana, R.; Brabec, C. J.; Heeger, A. J. Ultrafast Electron Transfer and Decay Dynamics in a Small Band Gap Bulk Heterojunction Material. Adv. Mater. 2007, 19, 2307-2312.

(32) Deibel, C.; Strobel, T.; Dyakonov, V. Role of the Charge Transfer State in Organic Donor-Acceptor Solar Cells. Adv. Mater. 2010, 22, 4097-4111.

(33) Bakulin, A. A.; Zapunidy, S. A.; Pshenichnikov, M. S.; Loosdrecht, P. H. M. v.; Paraschuk, D. Y. Efficient Two-Step Photogeneration of Long-Lived Charges in Ground-State ChargeTransfer Complexes of Conjugated Polymer Doped with Fullerene. Phys. Chem. Chem. Phys. 2009, 11, 7324.

(34) Veldman, D.; Meskers, S. C. J.; Janssen, R. A. J. The Energy of Charge-Transfer States in Electron Donor-Acceptor Blends: Insight into the Energy Losses in Organic Solar Cells. Adv. Funct. Mater. 2009, 19, 1939-1948.

(35) Zhou, Y.; Tvingstedt, K.; Zhang, F.; Du, C.; Ni, W.-X.; Andersson, M. R.; Inganäs, O. Observation of a Charge Transfer State in Low-Bandgap Polymer/Fullerene Blend Systems by Photoluminescence and Electroluminescence Studies. Adv. Funct. Mater. 2009, 19, 3293-3299.

(36) Tvingstedt, K.; Vandewal, K.; Gadisa, A.; Zhang, F.; Manca, J.; Inganäs, O. Electroluminescence from Charge Transfer States in Polymer Solar Cells. J. Am. Chem. Soc. 2009, 131, 11819-11824. 
(37) Tvingstedt, K.; Vandewal, K.; Zhang, F.; Inganäs, O. On the Dissociation Efficiency of Charge Transfer Excitons and Frenkel Excitons in Organic Solar Cells: A Luminescence Quenching Study. J. Phys. Chem. C 2010, 114, 21824-21832.

(38) Shoaee, S.; Clarke, T. M.; Huang, C.; Barlow, S.; Marder, S. R.; Heeney, M.; McCulloch, I.; Durrant, J. R. Acceptor Energy Level Control of Charge Photogeneration in Organic Donor/Acceptor Blends. J. Am. Chem. Soc. 2010, 132, 12919-12926.

(39) Devizis, A.; Serbenta, A.; Meerholz, K.; Hertel, D.; Gulbinas, V. Ultrafast Dynamics of Carrier Mobility in a Conjugated Polymer Probed at Molecular and Microscopic Length Scales. Phys. Rev. Lett. 2009, 103, 4.

(40) Vithanage, D. A.; Devižis, A.; Abramavičius, V.; Infahsaeng, Y.; Abramavičius, D.; MacKenzie, R. C. I.; Keivanidis, P. E.; Yartsev, A.; Hertel, D.; Nelson, J.et al. Visualizing Charge Separation in Bulk Heterojunction Organic Solar Cells. Nat. Commun. 2013, 4, 2334.

(41) Gélinas, S.; Rao, A.; Kumar, A.; Smith, S. L.; Chin, A. W.; Clark, J.; van der Poll, T. S.; Bazan, G. C.; Friend, R. H. Ultrafast Long-Range Charge Separation in Organic Semiconductor Photovoltaic Diodes. Science 2014, 343, 512-516.

(42) Melianas, A.; Pranculis, V.; Devižis, A.; Gulbinas, V.; Inganäs, O.; Kemerink, M. Dispersion-Dominated Photocurrent in Polymer:Fullerene Solar Cells. Adv. Funct. Mater. 2014, 24 , 4507-4514.

(43) Herrmann, D.; Niesar, S.; Scharsich, C.; Kohler, A.; Stutzmann, M.; Riedle, E. Role of Structural Order and Excess Energy on Ultrafast Free Charge Generation in Hybrid Polythiophene/Si Photovoltaics Probed in Real Time by near-Infrared Broadband Transient Absorption. J. Am. Chem. Soc. 2011, 133, 18220-18233.

(44) Falke, S. M.; Rozzi, C. A.; Brida, D.; Maiuri, M.; Amato, M.; Sommer, E.; De Sio, A.; Rubio, A.; Cerullo, G.; Molinari, E.et al. Coherent Ultrafast Charge Transfer in an Organic Photovoltaic Blend. Science 2014, 344, 1001-1005.

(45) Pensack, R. D.; Asbury, J. B. Ultrafast Probes of Charge Transfer States in Organic Photovoltaic Materials. Chem. Phys. Lett. 2011, 515, 197-205.

(46) Bakulin, A. A.; Lovrincic, R.; Yu, X.; Selig, O.; Bakker, H. J.; Rezus, Y. L. A.; Nayak, P. K.; Fonari, A.; Coropceanu, V.; Bredas, J.-L.et al. Mode-Selective Vibrational Modulation of Charge Transport in Organic Electronic Devices. Nat. Commun. 2015, 6, 7880.

(47) Bakulin, A. A.; Morgan, S. E.; Kehoe, T. B.; Wilson, M. W. B.; Chin, A. W.; Zigmantas, D.; Egorova, D.; Rao, A. Real-Time Observation of Multiexcitonic States in Ultrafast Singlet Fission Using Coherent 2d Electronic Spectroscopy. Nat. Chem. 2016, 8, 16-23.

(48) Jarzab, D.; Cordella, F.; Gao, J.; Scharber, M.; Egelhaaf, H.-J.; Loi, M. A. LowTemperature Behaviour of Charge Transfer Excitons in Narrow-Bandgap Polymer-Based Bulk Heterojunctions. Adv. Energy Mater. 2011, 1, 604-609.

(49) Mangold, H.; Bakulin, A. A.; Howard, I. A.; Kastner, C.; Egbe, D. A. M.; Hoppe, H.; Laquai, F. Control of Charge Generation and Recombination in Ternary Polymer/Polymer:Fullerene Photovoltaic Blends Using Amorphous and Semi-Crystalline Copolymers as Donors. Phys. Chem. Chem. Phys. 2014, 16, 20329-20337.

(50) Marsh, R. A.; Hodgkiss, J. M.; Friend, R. H. Direct Measurement of Electric FieldAssisted Charge Separation in Polymer:Fullerene Photovoltaic Diodes. Adv. Mater. 2010, 22, 36723676.

(51) Mingebach, M.; Walter, S.; Dyakonov, V.; Deibel, C. Direct and Charge Transfer State Mediated Photogeneration in Polymer--Fullerene Bulk Heterojunction Solar Cells. App. Phys. Lett. 2012, 100, 193302-193304.

(52) Wang, E.; Hou, L.; Wang, Z.; Hellström, S.; Zhang, F.; Inganäs, O.; Andersson, M. R. An Easily Synthesized Blue Polymer for High-Performance Polymer Solar Cells. Adv. Mater. 2010, 22, 5240-5244.

(53) Gao, F.; Himmelberger, S.; Andersson, M.; Hanifi, D.; Xia, Y.; Zhang, S.; Wang, J.; Hou, J.; Salleo, A.; Inganäs, O. The Effect of Processing Additives on Energetic Disorder in Highly Efficient Organic Photovoltaics: A Case Study on Pbdttt-C-T:Pc71bm. Adv. Mater. 2015, 27, 3868-3873. 
(54) Vandewal, K.; Tvingstedt, K.; Gadisa, A.; Inganas, O.; Manca, J. V. Relating the OpenCircuit Voltage to Interface Molecular Properties of Donor:Acceptor Bulk Heterojunction Solar Cells. Phys. Rev. B 2010, 81, 125204.

(55) Veldman, D.; İpek, Ö.; Meskers, S. C. J.; Sweelssen, J.; Koetse, M. M.; Veenstra, S. C.; Kroon, J. M.; Bavel, S. S. v.; Loos, J.; Janssen, R. A. J. Compositional and Electric Field Dependence of the Dissociation of Charge Transfer Excitons in Alternating Polyfluorene Copolymer/Fullerene Blends. J. Am. Chem. Soc. 2008, 130, 7721-7735.

(56) Bakulin, A. A.; Silva, C.; Vella, E. Ultrafast Spectroscopy with Photocurrent Detection: Watching Excitonic Optoelectronic Systems at Work. J. Phys. Chem. Lett. 2016, 7, 250-258.

(57) Frankevich, E.; Ishii, H.; Hamanaka, Y.; Yokoyama, T.; Fuji, A.; Li, S.; Yoshino, K.; Nakamura, A.; Seki, K. Formation of Polaron Pairs and Time-Resolved Photogeneration of Free Charge Carriers in P-Conjugated Polymers. Phys. Rev. B 2000, 62, 2505-2515.

(58) Muller, J. G.; Lemmer, U.; Feldmann, J.; Scherf, U. Precursor States for Charge Carrier Generation in Conjugated Polymers Probed by Ultrafast Spectroscopy. Phys. Rev. Lett. 2002, 88, 147401.

(59) Bakulin, A. A.; Neutzner, S.; Bakker, H. J.; Ottaviani, L.; Barakel, D.; Chen, Z. Charge Trapping Dynamics in Pbs Colloidal Quantum Dot Photovoltaic Devices. ACS Nano 2013, 7, 87718779.

(60) Pachoumi, O.; Bakulin, A. A.; Sadhanala, A.; Sirringhaus, H.; Friend, R. H.; Vaynzof, Y. Improved Performance of Zno/Polymer Hybrid Photovoltaic Devices by Combining Metal Oxide Doping and Interfacial Modification. J. Phys. Chem. C 2014, 118, 18945-11890.

(61) Provencher, F.; Bérubé, N.; Parker, A. W.; Greetham, G. M.; Towrie, M.; Hellmann, C.; Côté, M.; Stingelin, N.; Silva, C.; Hayes, S. C. Direct Observation of Ultrafast Long-Range Charge Separation at Polymer-Fullerene Heterojunctions. Nat. Commun. 2014, 5, 4288.

(62) Gao, F.; Tress, W.; Wang, J.; Inganäs, O. Temperature Dependence of Charge Carrier Generation in Organic Photovoltaics. Phys. Rev. Lett. 2015, 114, 128701. 
TOC graphics:

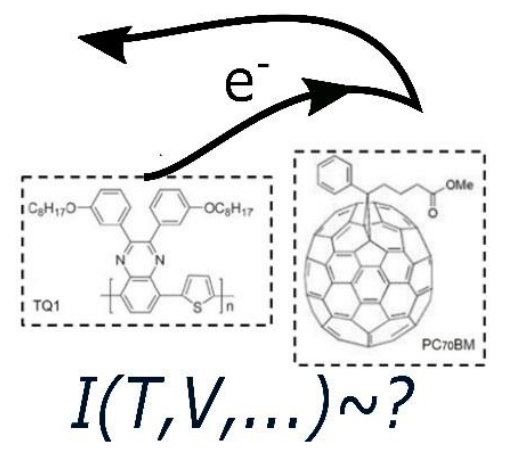

